against the posterior wall of the rectum when the patient is placed in the Fowler position. A straight hardrubber tube often causes considerable irritation, while the flexed tube causes no inconvenience. The rubber tube should always be strapped to the thigh. This insures its retention and prevents traction or pressure on the sphincter. Frequent change of the tube annoys the patient greatly and soon produces an irritability. 100 State Street.

\section{CONTINUOUS ENTEROCLYSIS}

\section{THE TWO-FUNNEL VISIBLE-DROP METHOD}

\section{SAMUEL E. NEWMAN, M.D.}

$$
\text { sT. LOUIS }
$$

There have been many attempts to simplify the method of giving a continuous saline solution into and most efficient method, has been discarded; "we can not make it work" being the usual excuse. All the substitutes that I have seen have been poor ones and unreliable makeshifts open to strong objections.

The simple device which is here offered meets every requirement necessary for the successful working of the method and possesses decided advantages.

1. The apparatus is elevated on the table at the side of the bed and need not be disturbed.

2. It is an open method, and any difficulty is easily. located.

3. The drops may be fed as fast or as slow as desirable, by means of an adjustment.

4. The drops are visible.

5. The rubber tube is not compressed and distention of the bowel is avoided; any return flow from the rectum is facilitated.

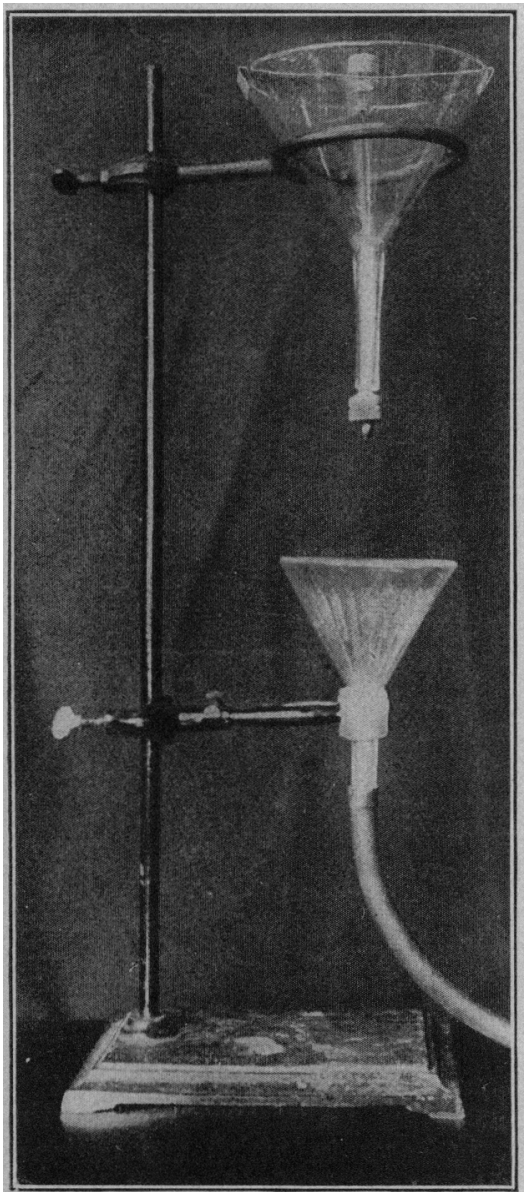

Fig. 1.-A laboratory stand with one large and one small fron ring; two glass funnels, the upper one large and the lower one small. A rubber tube attached to the lower funnel conveys the fluid to the rectum. The upper funnel is provided with a loosely fitting rubber plus at the tip and with a broad metal strip which is fastened across the top of the funnel. This metal strip has of the flut the middle and at thas an opening at the midale and at this point the nit of a screw is soldered in place. This seren rases or lowers the rubber which connects the two

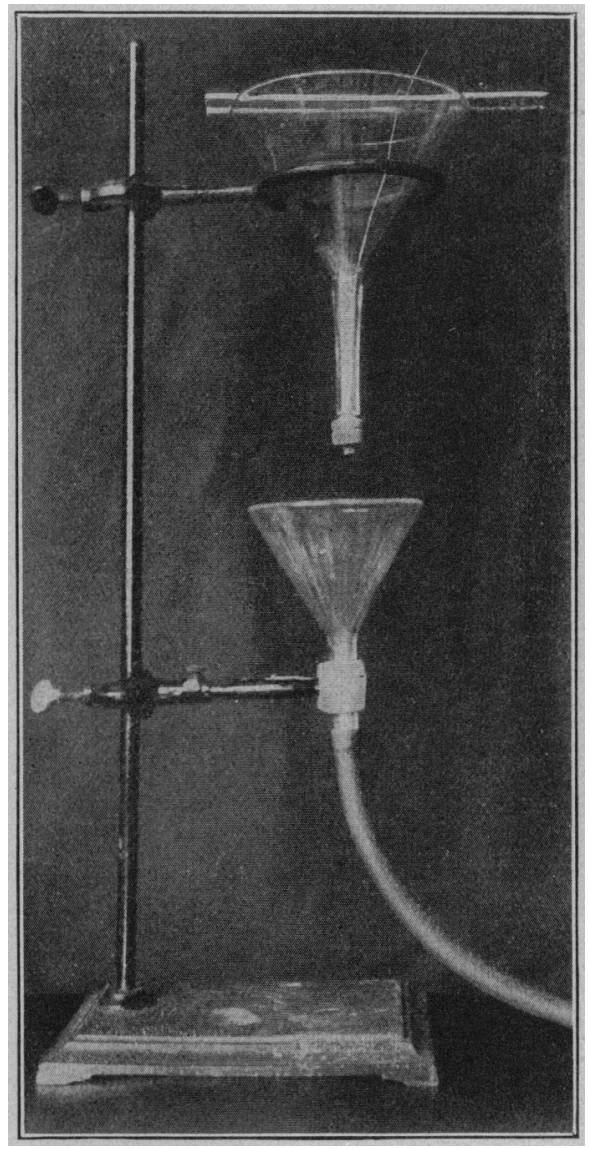

Fig. 2.-This is a home-made apparatus which can be easily devised. The upper funnel is provided with a rubber plug (the plunger of an antitoxin syringe has been used) to which a non-corroding wire is at tached by means of a small shot. Another small shot is fastened on the other end of the wire, and this is held in the groove of a small piece of wooden board. P'ushing the board toward the center of the funnel lengthens the wire and loosens the funcer plon while puling the little board from the tightens the fundel shortens the wire and tightens the rubber stopper. An incandes keep the saline solution from cooling.

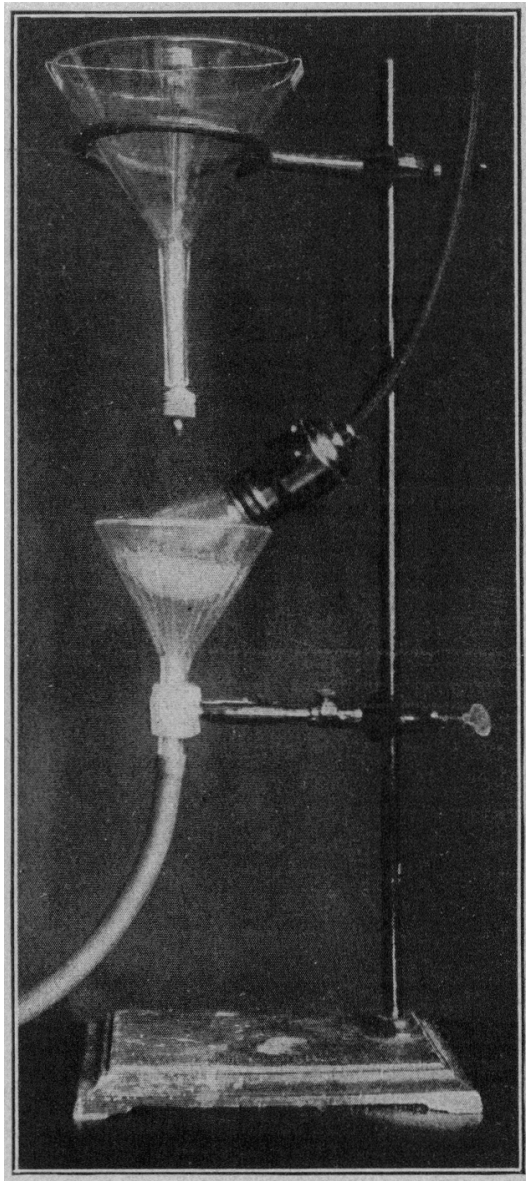

Fig. 3.-A porcelain evaporating-dish is placed in the lower funnel, and an electric bulb rests in this little cup. The upper funnel contains cool saline solution. As the water falls from the fun. nel above to the funnel below it strikes nel above to the funnel below it strikes
the electric bulb, flows down its sides and collects in the evaporating dish. It is warmed and gradually displaced by the additional solution which drops from above. The water does not become too warm unless the drops are made to fall at very long intervals. The heat must be tured of be turned off when the upper funnel is (hecked. the rectum, i. e., the Murphy treatment. Too much seems to depend on the close attention and frequert adjustment by the attendant. In many hospitals the original gravity plan, which is advisable as the simplest
6. Return flow from the rectum is seen and the drops may be fed slower or stopped entirely until the rectum has recovered itself.

I have attempted to solve the problem of maintain- 
ing the solution at a lukewarm temperature. In the majority of cases the water which the patient receives is cool or entirely too hot.

The apparatus shown in Figure 2 is arranged for hot saline solution, and in Figure 3 , a warm solution should be used in the upper funnel.

4900 Berlin Avenue.

\section{AN APPARATUS TO KEEP ENTEROCLYSIS SOLUTIONS HOT}

\section{B. B. WECHSLER, M.D.}

Ohstetrician to Montefiore Hospital PitTsBuir, PA.

This illustration shows an apparatus devised to keep at a proper temperature the solution for enteroclysis given by the Murphy drop method. This is difficult to accomplish by the usual method.

The apparatus consists of a glass irrigator encased in a jacket or nest made of copper for the outer wall and tin for the inner wall, having a space of half an inch between the outer and inner walls. An opening at the top fitted with an air-tight lid permits the filling

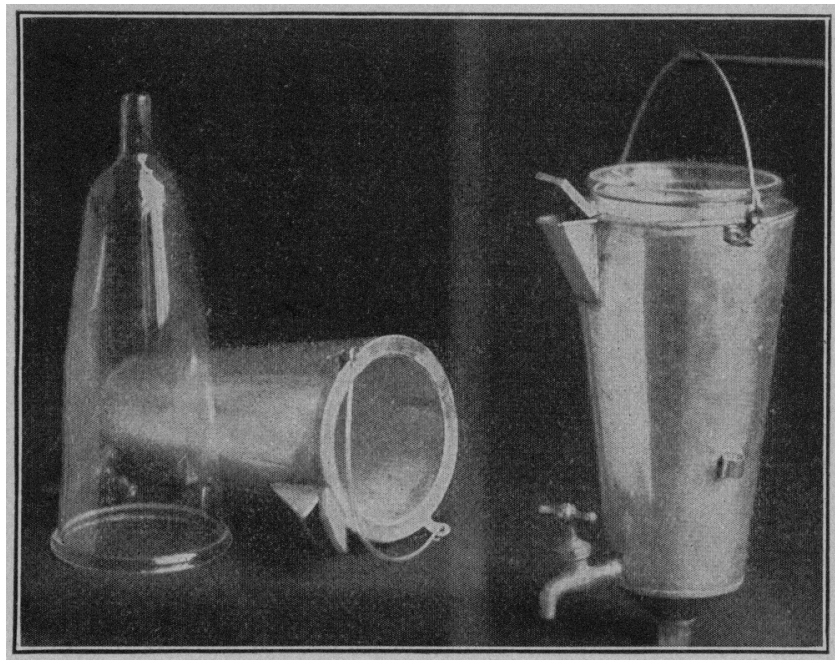

On the left, the glass irrigator and the jacket; on the right. the apparatus ready to use.

of the jacket with hot water, and a stop-cock at the base permits its removal when cold. The jacket is supplied with a handle for its support.

The irrigator protrudes about an inch at the base of the jacket, where the connection can be made. The apparatus is covered with a sterile towel when ready to be used.

Tests at the Montefiore Hospital of Pittsburg, Pa., showed the apparatus to work satisfactorily in every respect. The solution is put into the irrigator at a temperature of $130 \mathrm{~F}$. and hot water used as the heating medium. After three and one-half hours the solution is still at about $110 \mathrm{~F}$.

1401 Fifth Avenve.

Chorea Minor.--Forssner leans to the generally accepted opinion that the symptom-complex of chorea minor may be caused by various infections and possibly also other noxious influences. The results of his observations speak in favor of the tendency of the disease to attack especially individuals of feeble constitution. Many of those who in childhood suffered from chorea minor later acquire numerous chronic diseases.Hygica Festband.

\section{DISLOCATION OF THE KNEE AND DISLOCA-} TION OF THE FOOT

WALTER D. WISE, M.D. BALTIMORE

A case of anterior dislocation of the knee and a case of backward subastragaloid dislocation of the foot with fracture of the astragalus were both seen at the Baltimore City IIospital in the service of Dr. A. C. Harrison, and are reported because of the rarity of the conditions.

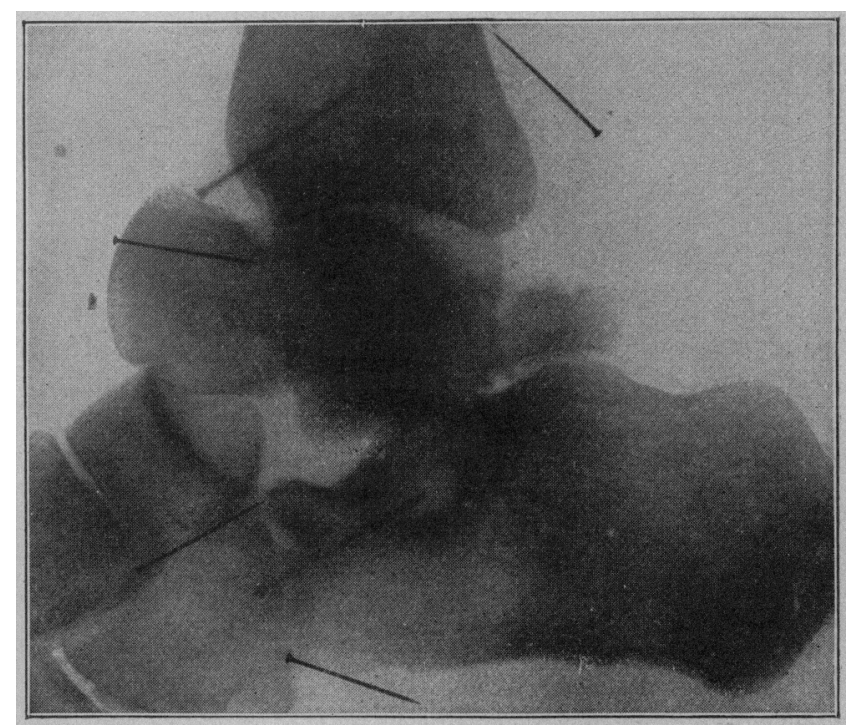

Fig. 1.-Dislocation of the astragaloscaphoid joint and fracture of the posterior part of the astragalus.

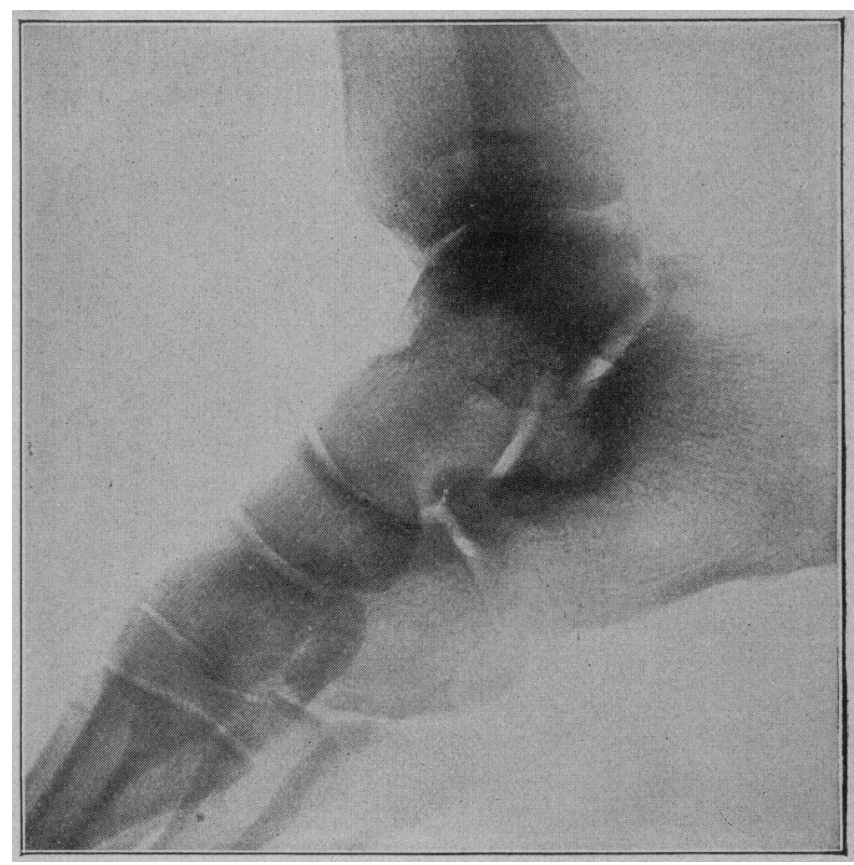

Fi- 2.-Dislocation reduced.

Dislocations of the knee compose less than 2 per cent. of all dislocations. Of these about one-half are of the forward variety, and 20 per cent. of this type are compound. Up to this time there have been reported 270 cases of knee dislocations including all varieties, 113 of these being of the anterior type, this making the one hundred and fourteenth case to be reported. 